\title{
Bizarre repetitive discharges recorded with single fibre EMG
}

\author{
JOŽE TRONTELJ, ERIK STÅLBERG \\ From the Institute of Clinical Neurophysiology, University Medical Centre, Ljubljana, Yugoslavia and the \\ Department of Clinical Neurophysiology, University Hospital, Uppsala, Sweden
}

SUMMARY Single fibre EMG was used to record bizarre repetitive discharges in patients with chronic denervation or muscle disorders. The low variability of intervals between individual spike components on successive discharges suggests that the bizarre repetitive discharges are based on ephaptic impulse transmission from the muscle fibre starting the discharge (principal pacemaker) to the adjacent muscle fibres. The low variability of the interdischarge intervals is explained by ephaptic reactivation of the principal pacemaker by one of the fibres participating in the previous discharge (a co-pacemaker). Secondary activator fibres may spread activity to more fibres. The bizarre repetitive discharge stops when either the principal pacemaker or the co-pacemaker become sufficiently subnormal to block. The co-pacemaker may be missing in cases of low frequency bizarre repetitive discharges, particularly in those with irregular interdischarge intervals.

Bizarre repetitive discharges represent a phenomenon commonly recorded with needle electrodes in clinical EMG studies of chronically denervated muscles, as well as in various myopathies. They are also known as bizarre high frequency discharges, complex repetitive discharges or pseudomyotonic discharges. ${ }^{1-8}$ In concentric needle EMG they most often appear as continuous trains of spikes of simple or complex patterns repeated at a regular frequency $(5-150 \mathrm{~Hz})$, and have an abrupt onset and termination. They can often be triggered by movement of the recording electrode. In addition to these discharges, low frequency bizarre discharges occurring at rates from 0.3 to $10 \mathrm{~Hz}$ have been described as a separate variety. ${ }^{9}$ It has been emphasised that these discharges have nothing in common with discharges in myotonic disorders. ${ }^{6}$

The present study was undertaken with single fibre EMG. The advantages of this method as compared to the concentric needle EMG include the high resolution with which the time intervals both within and between the discharges can be measured, as well as a better possibility of identifying individual muscle fibres participating in the different discharges.

Address for reprint requests: Stålberg, Department of Clinical Neurophysiology, University Hospital, 75014 Uppsala, Sweden.

Received 10 September 1982. Accepted 20 October 1982

\section{Patients and methods}

Sixteen patients volunteered to participate in the study: 10 with chronic partial or complete denervation due to peripheral nerve or root lesion, one with syringomyelia, two with Duchenne muscular dystrophy and three with polymyositis. Single fibre EMG (SFEMG) recordings were made from muscles which were completely denervated or weak due to partial denervation or myopathic involvement. The patient with syringomyelia had completely denervated biceps brachii muscle of one arm which showed unusually rich bizarre repetitive discharge activity, even resulting in a clinically visible tonic contraction. In this patient, the bizarre repetitive discharges were also recorded with a subcutaneously positioned concentric needle electrode, as well as with a pair of surface electrodes placed over the biceps brachii muscle. The recording technique has been described in detail elsewhere. ${ }^{10}$

\section{Results}

Several hundreds of recording sites with bizarre repetitive discharges have been studied. Only those lasting several seconds or more were analysed in detail. The bizarre repetitive discharges were usually spontaneous, and could be recorded with subcutaneously placed needle electrodes or with surface electrodes in a patient with syringomyelia over the biceps brachii which was totally denervated. Often the bizarre repetitive discharges were triggered by movements of the recording electrode or by percussion of the muscle. In the dystrophic muscles they could be initiated by voluntary activity of some motor units discharging at high 


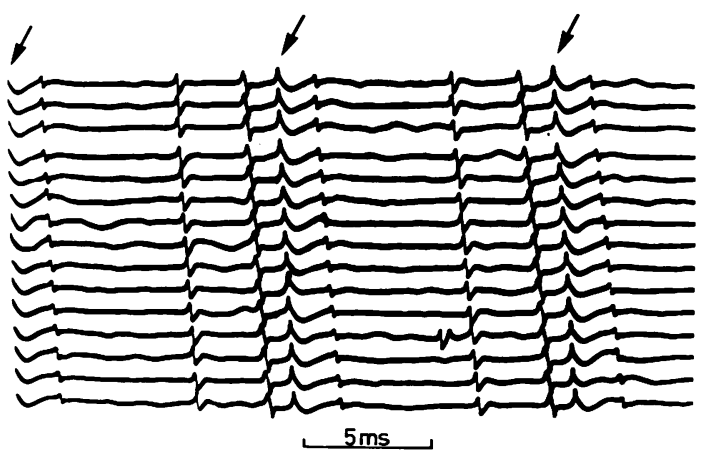

Fig 1 A bizarre repetitive discharge, recorded in a denervated muscle, consisting of four different components, one of which is a positive sharp wave, marked with an arrow (positivity is upwards in this recording). Notice pronounced progressive decline in the repetition. The beginning and the end of the basic discharge cannot be defined, but the duration of the cycle and the IDI can be measured between two successive spikes of identical fibres.

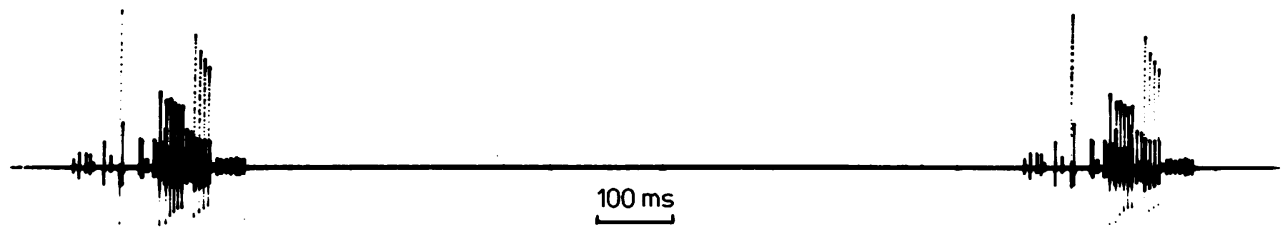

Fig $2 A$ bizarre repetitive discharge with a multispike basic complex occurring at a low and regular repetition rate (about $0.8 \mathrm{~Hz}$ ). The recording is from a patient with an advanced stage of Duchenne muscular dystrophy (cffig 4).

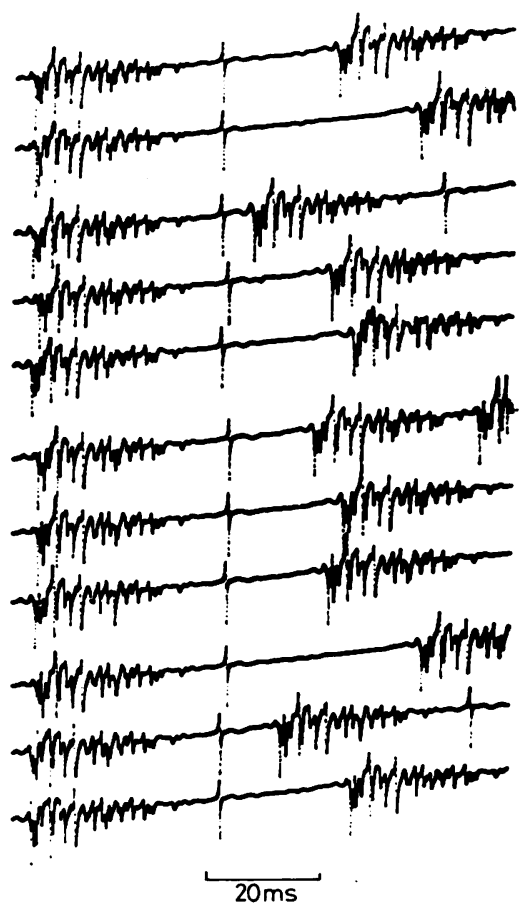

Fig 3 A bizarre repetitive discharge with a relatively low and very irregular repetition rate $(15$ to $25 \mathrm{~Hz}$ ). The recording is from a patient with a chronic root lesion. innervation rates. When a multispike voluntary discharge suddenly became transformed into a bizarre repetitive discharge the firing rate of the previously active fibres increased, their order of appearance changed and some of the fibres blocked. In one of the recordings each basic complex contained a positive sharp wave which was believed to trigger the bizarre repetitive discharge (fig 1).

The bizarre repetitive discharges lasting at least some seconds tended to show three different phases. The first was a short initial unstable phase, characterized by the presence of varying numbers of spikes occurring sometimes at changing intervals or even in a changing order, some of which disappeared after a few discharges. This was followed by a stable intermediate phase, when most or all of the components appeared very regularly. Finally there was a usually brief terminal phase with intermittent dropping out of individual components and occasionally with slowing of the discharge rate. This phase could be missing.

In our recordings the bizarre repetitive discharges consisted of at least two but usually more different spikes making up the so called basic complex. Each repetition of the basic complex is called a cycle. The repetition rate was from about $0.5 \mathrm{~Hz}$ to more than $100 \mathrm{~Hz}$. When the rate was high it was sometimes difficult to define the beginning and the end of the basic complex (fig 1). Nevertheless the discharges of individual muscle fibres could be well recognised from the shape of the action potentials and thus the repetition rate and the duration of the basic complex could be measured. The interval between two consecutive basic complexes is called interdischarge interval (IDI); it could be measured between any two successive spikes of identical fibres. The variability of IDI was calculated as the 

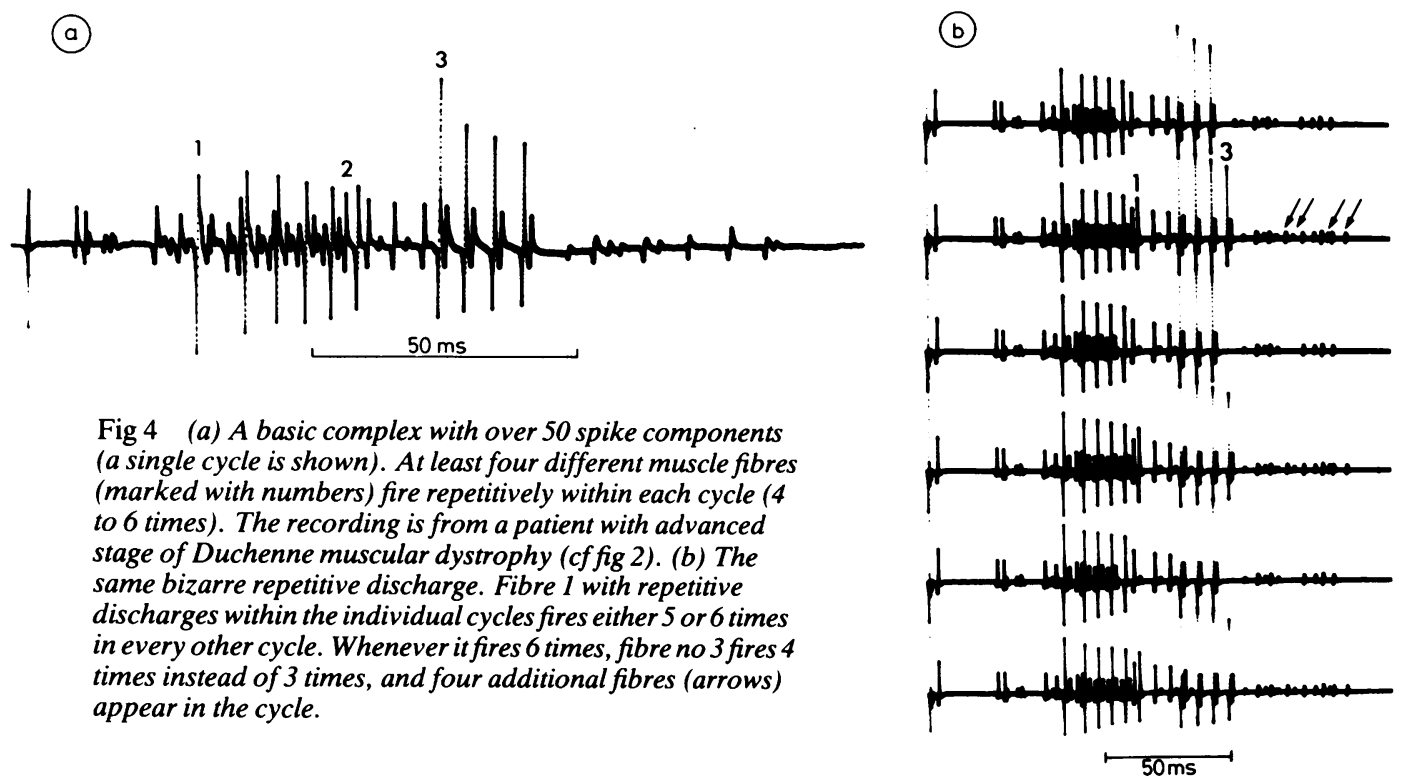

Fig 4 (a) A basic complex with over 50 spike components (a single cycle is shown). At least four different muscle fibres (marked with numbers) fire repetitively within each cycle (4 to 6 times). The recording is from a patient with advanced stage of Duchenne muscular dystrophy (cffig 2). (b) The same bizarre repetitive discharge. Fibre 1 with repetitive discharges within the individual cycles fires either 5 or 6 times in every other cycle. Whenever it fires 6 times, fibre no 3 fires 4 times instead of 3 times, and four additional fibres (arrows) appear in the cycle.

mean of consecutive interval differences (IDI-jitter). The IDI-jitter could be lower than 2-5 $\mu$ s but was often larger. It could be considerably larger in the initial and terminal unstable phases. There were often slow trends in the IDI with an increase as the bizarre repetitive discharge approached its abrupt termination. Rarely every second or every third basic complex was dropping out.

Bizarre repetitive discharges with low repetition rates, for example below $20 \mathrm{~Hz}$, were less often encountered (fig 2). Then the IDI was occasionally highly irregular (fig 3 ). In other respects there were no principal differences between the high and low frequency bizarre repetitive discharges.

The basic complex typically contained two to more than 50 spike components. The individual spikes were usually well spaced, sometimes with long interpotential intervals, less often partly superimposed on one another. Duration of the basic complex was often more than $20 \mathrm{~ms}$ and up to 170 ms. The spike components most often seemed to belong to different muscle fibres as indicated by their difference in shape and independent changes in amplitude on slight 


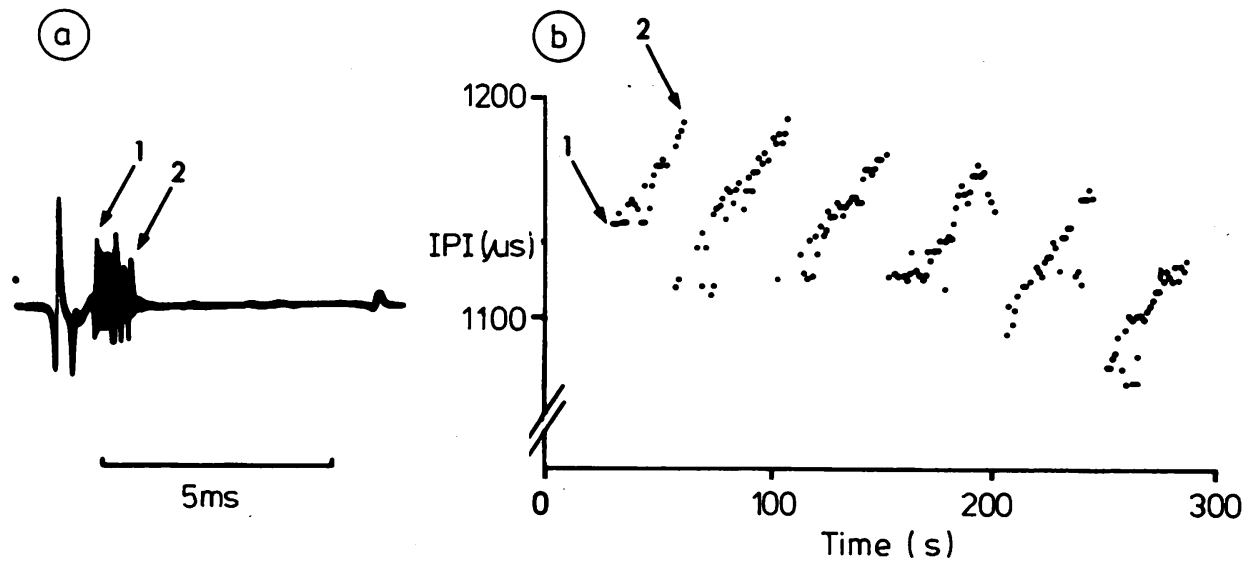

Fig 6 One of the spikes in the basic complex shows gradually increasing IPI followed by intermittent blocking. An increased jitter (between arrows) is seen in the superimposed recording of about 20 cycles. (a) The sequence is repeated several times, as shown in sequential IPI histogram in. (b) A recording from a partially denervated muscle.

(a)
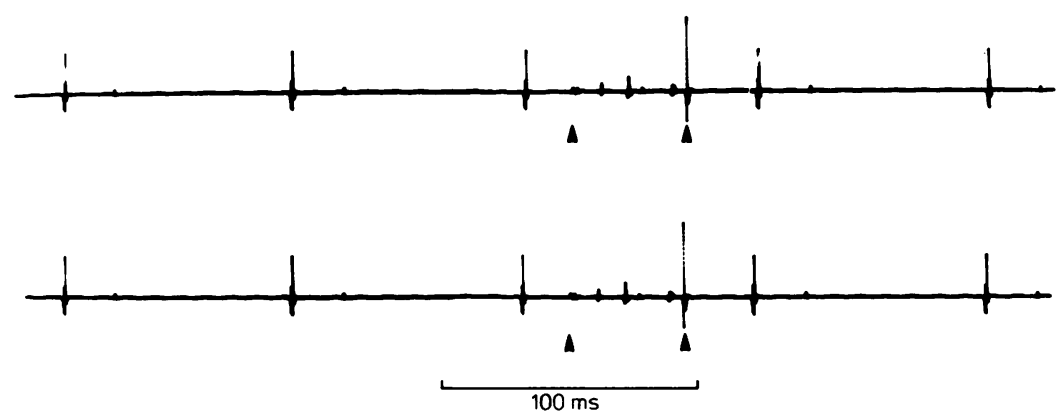

Fig 7 (a) Continuous recording from a denervated muscle showing intermittent blocking of a group of muscle fibres (between arrows) in a bizarre repetitive discharge. The blocking group of muscle fibres appeared time locked to the regularly firing pair of spikes but only occurred in one out of 5 cycles. (b) Temporary blocking of two muscle fibres (marked with circles), obviously driven by a third fibre (marked with a star). In the trace marked with the lower arrow, this fibre suddenly gave a brief burst resulting in profound subnormality (evident as change in potential shape and amplitude) and block, associated with dropping out of the two dependent fibres. Seven traces later (upper arrow) when excitability of the secondary activator fibre is recovered the whole group reappears in the bizarre repetitive discharge. (b)

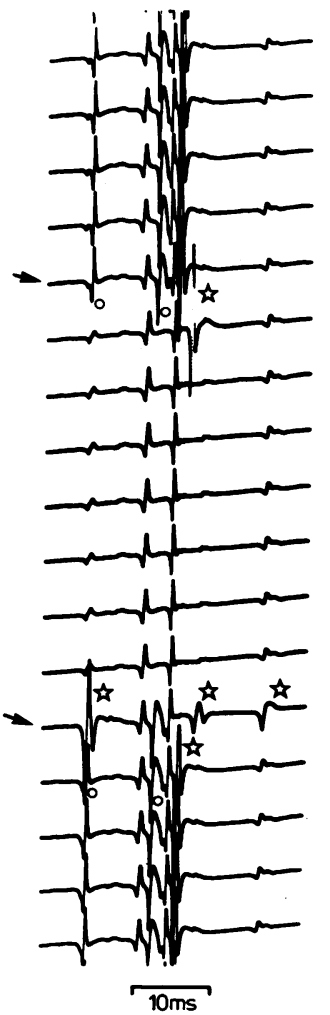




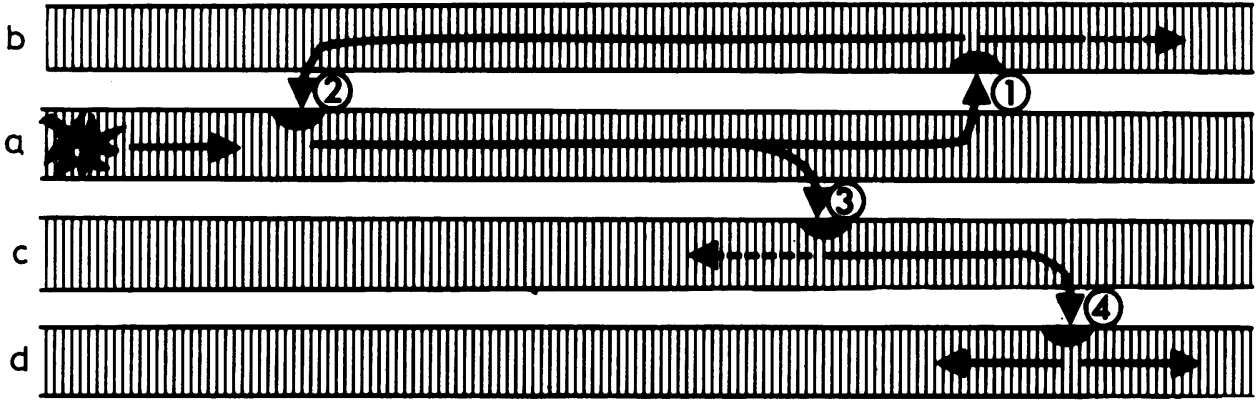

Fig 8 Schematic drawing of the suggested mechanism of bizarre repetitive discharges. a: principal pacemaker fibre, $b$ : co-pacemaker fibre. c: secondary activator; $d$ : a fibre dependent on the secondary activator. The site of initiation of a fibrillation potential starting the bizarre repetitive discharge is marked with a spark. The low threshold sites at which ephaptic transmission occurs are indicated as black areas. Transmission block at sites 1 or 2 will give a low frequency bizarre repetitive discharge (an open loop bizarre repetitive discharge) or stop the bizarre repetitive discharge altogether. Block at site 3 will result in concomitant, block at site 4 in a single spike blocking.

movement of the recording electrode. Sometimes, however, the same fibre generated a train of spikes within the basic complex. This was evident from their decreasing amplitude and recovery after regular pauses (fig 4) or after drop out of individual discharges (fig 5).

During the stable intermediate phase of the bizarre repetitive discharge sequence the jitter between individual spikes (the IPI-jitter) was typically less than $5 \mu \mathrm{s}$. In the initial and terminal phases this jitter could be much larger and associated with intermittent drop out of the most unstable components (fig 6). The drop out could be quite regular, for example one out of five discharges, and as a rule gradually became more frequent. Sometimes the degree of drop out decreased during the bizarre repetitive discharge. Occasionally two or several spike components showed a concomitant jitter and blocking (fig 7).

\section{Discussion}

The site of origin and the way of spreading of bizarre repetitive discharges have been questioned by earlier workers. Nerve blocks and curare failed to abolish these discharges. ${ }^{79}$ It was concluded therefore that they are started in the muscle fibres and in some way extraneurally transmitted to neighbouring fibres.

Bizarre repetitive discharges are considered a nonspecific finding seen both in neurogenic disorders and in myopathies, in normal urethral sphincter muscle "1 and in Schwartz-Jampel syndrome seen by us and also reported by others. ${ }^{12}$ Recently one of us (ES) had the opportunity to study five horses referred with cramps as the only clinical feature. EMG showed typical bizarre repetitive discharges that persisted after a paralysing dose of curare. The present study did not reveal any significant difference between the bizarre repetitive discharges recorded in the different disorders and are therefore discussed together.

Single fibre EMG used in the present study allowed a reliable identification of individual muscle fibre action potentials, as well as precise measurement of the IDI and IPI-jitter. An important finding is the low jitter, that is less than $5 \mu \mathrm{s}$, between the individual spike components, as well as between consecutive discharges. Such a low jitter is never obtained when an impulse transmission occurs across a motor endplate, as for instance in intramuscular nerve stimulation. Thus the finding of low jitter is interpreted as evidence of direct electrical, that is ephaptici activation. The following sequence of events is proposed in the generation of a bizarre repetitive discharge (fig 8).

The discharge is initiated by a spontaneously fibrillating muscle fibre, the principle pacemaker fibre. This fibre ephaptically activates one or several adjacent muscle fibres, when its action potential passes low threshold sites scattered along their membrane. One of these muscle fibres in turn ephaptically re-activates the principal pacemaker, which is thus discharged earlier than it would fire by itself. This fibre is called the co-pacemaker (in our earlier reports co-principal pacemaker or accelerator $\left.{ }^{10} 11^{13}\right)$. A closed loop is thus created. The impulse conduction time through the loop will determine the cycle time and the repetition rate. Ephaptic reactivation of the principal pacemaker fibre explains the low IDI-jitter while short loops account for the relatively high repetition rates.

On the other hand, all of the bizarre repetitive discharges do not have a low IDI-jitter. This is particularly true of the bizarre repetitive discharges with low repetition rates where the interdischarge intervals may even be highly irregular (see fig 3 ). It is believed that in these cases the co-pacemaker fibre is missing and thus the loop remains open. Then the bizarre repetitive discharge exclusively depends on 
the firing rhythm of the principal pacemaker fibre, which is correlated to the time course of the supernormal excitability of the site of impulse initiation. The IDI-jitter will necessarily be larger, for example similar to that of fibrillation potentials. Exceptionally even a closed loop bizarre repetitive discharge may show a high IDI-jitter, owing to threshold ephaptic transmission from the copacemaker to the principal pacemaker. This however can only occur for a short period of time, for example in the terminal unstable phase.

The bizarre repetitive discharge will last until either the principal pacemaker or the co-pacemaker fibre have become sufficiently subnormal to block. This will then result in an abrupt termination of the whole bizarre repetitive discharge. The increasing subnormality may be recognised from slightly increased IDI and IPI jitter just a few cycles before the end of the bizarre repetitive discharge. Another possible cause of termination of a bizarre repetitive discharge might be an extra-discharge from another generator site, blocking the impulse in the loop by collision.

Within the basic complex, some fibres may serve as secondary activators, each recruiting one or more additional fibres. This explains the occasional drop out from the basic discharge of a group of spikes which may immediately before show an increased jitter (concomitant jitter and blocking), evidently due to increasing subnormality and to rising threshold of the secondary activator. The phenomenon of repetitive discharges of individual muscle fibres within each individual cycle is probably due to repetitive extra-discharges ${ }^{1014}$ in one fibre. Such extra-discharges could even act as secondary activators as shown in fig 4 .

The occasional finding of a drop out of, say every second or third cycle, can be interpreted as blocking of a secondary activator with its dependent fibres, the principal pacemaker and the co-pacemaker fibres being too remote to be recorded by the microelectrode.

The IPI jitter between the different spikes in the basic complex was typically low, that is less than $5 \mu \mathrm{s}$. Occasionally, one or several spikes could show gradually increasing jitter followed by intermittent blocking at regular, but increasing rates. This is again interpreted as a result of increasing subnormality of the site of ephaptic transmission. The blocking spike might have no influence on the firing rate of the basic complex. Sometimes however such single spike blocking might, after just a few more cycles, be followed by termination of the whole discharge. This may indicate that even such fibres, evidently not entirely indispensible for the bizarre repetitive discharge, may nevertheless support it, possibly by providing subdepolarisation of either the principal pacemaker or the co-pacemaker fibre.

Duration of the basic complex, which was most often between 10 and $50 \mathrm{~ms}$, was in the average longer than the multiple spike recordings that could be obtained during voluntary activation in chronic partial denervation and even in dystrophic muscles. This long duration of the bizarre repetitive discharges could be due to scattering of the low threshold sites along the muscle fibres, which function as sites of ephaptic transmission. Another possible explanation would be slow conduction in atrophic muscle fibres, giving rise to long loop time.

The presence of the needle electrode as a mechanical stimulator is evidently not needed for the generation of bizarre repetitive discharges as they could be recorded with a subcutaneously placed needle electrode or with surface electrodes and were seen to start in the absence of any obvious mechanical or other stimulus, giving rise to cramps in the completely denervated biceps brachii muscle of the patient with syringomyelia. Such cramps also occur in patients with amyotrophic lateral sclerosis. On the other hand, mechanical stimulation may provoke bizarre repetitive discharges, as is known from clinical EMG. Figure 1 shows such a case where a positive sharp wave was elicited by the movement of the electrode and took part in a bizarre repetitive discharge; conceivably it could have acted as a pacemaker for the whole discharge.

In a dystrophic muscle, bizarre repetitive discharges may have started in presumably normally innervated muscle fibres. This has been seen to occur when voluntary discharges exceeded certain frequency. Then the previously voluntary discharging fibres took up an even higher firing rate, either resulting in a higher repetition rate of the basic discharge or in repetitive extra-discharges within the individual cycles. Sites with abnormally low depolarisation threshold, which is even decreased during high frequency voluntary discharges are probably responsible for the initiation of the bizarre repetitive discharge.

The high amplitudes of individual spike components in bizarre repetitive discharges seem to speak against substantial atrophy of these fibres. Whether this means that the same fibres participate in bizarre repetitive discharges for a considerable time which prevents atrophy or indicates that bizarre repetitive discharges are more likely to occur in larger fibres is so far not known.

This work was supported by the Research Council of Slovenia, Grant No 90 (JT) and by the Swedish Medical Research Council, Grant No 135 (ES). 


\section{References}

' Adrian ED, Geifan S. Rhythmic activity in skeletal muscle fibres. J Physiol (Lond) 1933;78:271-86.

${ }^{2}$ Brumlik J, Cuetter C. Denervation myotonia: a subclinical electromyographic finding. Electromyography 1969;9:297-310.

${ }^{3}$ Brumlik J, Drechsler B, Vannin TM. The myotonic discharge in various neurological syndromes: a neurophysiological analysis. Electromyography 1970; 10:369-83.

${ }^{4}$ Emeryk B, Hausmanova-Petrusewicz J, Nowak T. Spontaneous volleys of bizarre high frequency potentials in neuromuscular diseases. Part 1: Occurrence of spontaneous volleys of bhfp in neuromuscular diseases. Electromyogr Clin Neurophysiol 1974;14:303-12.

${ }^{5}$ Kugelberg E, Petersen I. "Insertion activity" in electromyography. J Neurol Neurosurg Psychiatry 1949;12:268-73.

${ }^{6}$ Ludin HP. Electromyography in Practice. New York: Thieme-Stratton, 1980.

${ }^{7}$ Ricker K, Meinck HM. Discharge pattern and origin of "pseudomyotonic" (high frequency) discharge trains in denervation syndromes. Z. Zeitschrift für Elektroenzephalographie Elektromyographie und verwandte Gebiete (Thieme, Stuttgart) 1972;3:170-8.

${ }^{8}$ Ruprecht EO. Befunde bei Neuropathien. In: Elektromyographie Hrsg. von H.C. Hopf u. A. Struppler, Stuttgart: Thieme 1974.

9 Stoehr M. Low frequency bizarre discharges. Electromyogr Clin Neurophysiol 1978;18:147-56.

${ }^{10}$ Stålberg E, Trontelj JV. Single fibre electromyography. Mirvalle Press, Old Woking, Surrey 1979.

" Stålberg E, Trontelj JV. Abnormal discharges generated within the motor unit as observed with single fibre electromyography. In: Culp W and Ochoa J, eds. Abnormal nerves and muscles as impulse generators. Oxford: Oxford University Pre:s. 1982:443-74.

${ }_{12}$ Jablecki C. Single muscle fibre recordings in SchwartzJampel syndrome. (Symposium to honor EH Lambert). Muscle Nerve, 1983 (in press).

${ }^{13}$ Trontelj JV. Ephaptic transmission in the human skeletal muscle. Zdrav Vestn 1980;49:181.

${ }^{14}$ Trontelj JV, Stålberg E. Response to electrical stimulation of denervated human muscle fibres recorded with single fibre EMG. J Neurol Neurosurg Psychiatry 1983;46:305-9. 